\title{
De-noising of partial discharge signal using wavelet transform for GIS under HVDC
}

\author{
Guoming Wang ${ }^{1}$, Han $\mathbf{H u}^{1}$, Min Tang ${ }^{1}$, Jinlong Gong ${ }^{2}$, \\ Yifan Wang ${ }^{2}$, Hong-Keun $\mathrm{Ji}^{3}$, Gyung-Suk Kil ${ }^{4}$
}

\begin{abstract}
Detection and analysis of partial discharge (PD) have been regard as the most effective method for condition monitoring and asset management of gas-insulated structures (GIS) in the power system. However, PD detection sensitivity and accuracy are greatly influenced by on-site noise and interference, resulting in failures in PD severity assessment, defect identification or localization. Although de-noising of PD signal under AC was well studied, related investigations under DC have not been carried out. With the rapid development of HVDC technology, it is a new challenge to eliminate noise from PD signal under DC for diagnosis of related power facilities. Therefore, this paper dealt with the discrimination of PD signal based on wavelet transform (WT) techniques for HVDC GIS, aiming to improve the sensitivity and accuracy of insulation diagnosis. Experimental setup was configured to generate PD signal under DC and four types of artificial defects were fabricated to simulate typical insulation defects in GIS. The WT techniques were used to discriminate PD pulse sequences from background noise, amplitude modulation radio interference, non-sinusoidal noise, and switching impulse and the effectiveness was compared with a high-pass filter.
\end{abstract}

K e y w or d s: discharge, de-noising, wavelet transform, gas-insulated structures, HVDC

\section{Introduction}

Failures of electrical power facilities are primarily resulted from poor insulation condition, causing serious economic, safety, and environmental issues. Condition monitoring and diagnosis are important methods to detect the insulation degradation at its early state and to ensure the reliability of power apparatuses. The diagnostic strategies for power facilities have been developed from run-to-failure, through maintenance as necessary, timebased maintenance, and condition-based maintenance, to the reliability-centered maintenance (RCM) that is advocated nowadays. The RCM strategy is a combination of the reactive, preventive, predictive, and proactive maintenance to maximize the reliability and availability of power apparatus [1-3]. The partial discharge (PD), which is a localized electrical discharge that partially bridges the insulation, causes progressive insulation deterioration and finally results in the failure of power equipment. Therefore, detection and characterization of $\mathrm{PD}$ on-site and on-line are important parts of RCM. Based on the measurement of PD, insulation defects can be detected before the eventual breakdown, and therefore the reliability and availability of equipment can be significantly enhanced and the unplanned outage of power system can be decreased, making the operation intervals extend and the maintenance cost reduce. The safety of asset manager and service personnel can be also ensured owning to less risk of explosion and combustion accidents. In addition, the environmental risk such as leakage of harmful gas and greenhouse gas can be reduced [4].

As a main indicator of electrical failure, detection, analysis, classification, and localization of PD under alternative current $(\mathrm{AC})$ have been well studied [5-10]. Based on the different physical and chemical phenomena accompanying with $\mathrm{PD}$, detection methods can be categorized into conventional method according to IEC 60270 and non-conventional methods, including the acoustic emission, ultra-high frequency, optical detection, and dissolved gases methods. Generally, physical PD signals are analyzed in time or frequency domain, such as the single pulse, phase-resolved $\mathrm{PD}$, time-resolved $\mathrm{PD}$ as well as time-frequency method, and the dissolved gases are analyzed in terms of gas ratios. The parameters extracted from various patterns can be used for PD identification, which is realized by machine learning algorithms like the artificial neural network, fuzzy logic, and supported vector machine. In addition, PD source can be localized using the absolute and relative time of arrival of acoustic emission or ultra-high frequency signal. However, owing to the absence of phase information and the different recurrence mechanism, PD analysis methods under direct current (DC) are totally different from those under AC, although the detection methods are the same [11-13]. The pulse repetition rate and frequency under $\mathrm{DC}$ are much lower than those under AC [14]. As a result, parameters for PD classification are also different. Until now, PD under DC has not been studied in detail, and experience from

\footnotetext{
${ }^{1}$ Hangzhou Guozhou Power Technology Co., Ltd., Hangzhou 310015, China, guoming_wang@hotmail.com, 2 State Grid Zhejiang Electric Power Research Institute, State Grid Zhejiang Electric Power Co., Ltd, Hangzhou 310014, China, gong_jinlong@zj.sgcc.com.cn, ${ }^{3}$ Forensic Safety Section, National Forensic Service Busan Institute, Yangsan 50612, Korea, hkji1024@korea.kr, ${ }^{4}$ Department of Electrical and Electronics Engineering, Korea Maritime and Ocean University, Busan 49112, Korea, hvlab@kmou.ac.kr
} 
PD under AC cannot be applied to DC directly, including the de-noising method. As the high voltage direct current (HVDC) distribution and transmission systems increase rapidly over the world, it is necessary to deal with $\mathrm{PD}$ under DC voltage for the purpose of risk assessment and asset management of related power facilities.

The gas-insulated structures (GIS) such as switchgear and transmission line are filled with Sulphur Hexafluoride (SF6) gas and have high insulation and arc-extinguishing capability, ensuring their high reliability, compact size, and economical efficiency [15-16]. As a result, they are widely installed in space-limited locations, such as city substations, offshore plants, and electric railway systems. The gas insulated switchgear, which is composed of circuit breaker, disconnect switch, grounding switch, and other high voltage components, has been used for power transmission and distribution sine 1960s. In addition, gas insulated transmission line is regarded as a safe and flexible alternative to an overhead line. However, insulation defects generated during the manufacture, assembly, transportation, and operation are one of the primary causes of GIS failures. Owing to the large power supply capacity, these failures result in enormous economic losses and serious human injuries. Therefore, diagnostics of PD are implemented for condition monitoring of GIS to ensure their reliable operation.

$\mathrm{PD}$ is evaluated using apparent charge that is expressed in picocoulomb $(\mathrm{pC})$. It is specified that the maximum permissible PD level for GIS should not exceed 5 pC [17]. However, when PD signals are buried in excessive noise or interference, they cannot be exactly discriminated, leading to a reduce in detection sensitivity and a failure of assessing PD severity. Furthermore, noise is a major bottleneck that influences the accuracy of defect identification and localization. In practice, the on-site noise and interference can be classified as:

- White noise from amplifier and ambient noise, which is a random signal with equal intensity at different frequencies.

- Discrete spectral interferences (DSI) from communication system, frequency modulation (FM) radio, and amplitude modulation (AM) radio, whose frequency bands are $890 \mathrm{MHz}-1.88 \mathrm{GHz}, 88 \mathrm{MHz}-108 \mathrm{MHz}$, and $535 \mathrm{kHz}-1605 \mathrm{kHz}$, respectively. The communication interference and FM radio have frequencies much higher than that of PD electrical current pulse, therefore, only the AM radio interference is taken into consideration when the conventional PD detection method is used.

- Periodic non-sinusoidal noise from power electronics or other periodic switching operations.

- Stochastic pulse-shaped interferences from infrequent switching operation, arcing between adjacent metallic contacts, and corona emitted from high voltage equipment [18-19].

Although great efforts have been made to suppress the noise associated with PD measurement, the existing methods have inherent limitations. The balance circuit recommended in IEC 60270 requires addition experiment devices and is not suitable for on-line application [5]. The fast Fourier transform (FFT) only analyzes signals in frequency domain. Since actual PD pulses are non-periodic, transient, and irregular, and frequencies of various noises are not easily to be determined, the FFT method has difficulty in deciding the threshold [19]. When the digital filter is implemented, there is also difficulty in determining the optimal cutoff frequency. As a result, the noises cannot be completely removed and original PD signal may be distorted [20].

Wavelet transform (WT), which was first introduced in practical application in mid-1980s, analyzes signals in both time and frequency domain simultaneously, and has been recognized as an effective method to discriminate PD from noise. Previous works have been done to reject noises from PD under AC [18-22] whereas there is few related research so far dealing with PD under HVDC. Therefore, this paper dealt with the de-noising of PD signal based on the WT for HVDC GIS, aiming to improve the detection sensitivity and accuracy.

\section{Experimental setup}

Configuration of experimental setup is illustrated in Fig. 1. A HVDC source was generated by a rectifier circuit that was composed of a dry-type transformer, a $100 \mathrm{kV}$ diode, and a $0.5 \mu \mathrm{F}$ capacitor. The transformer with a

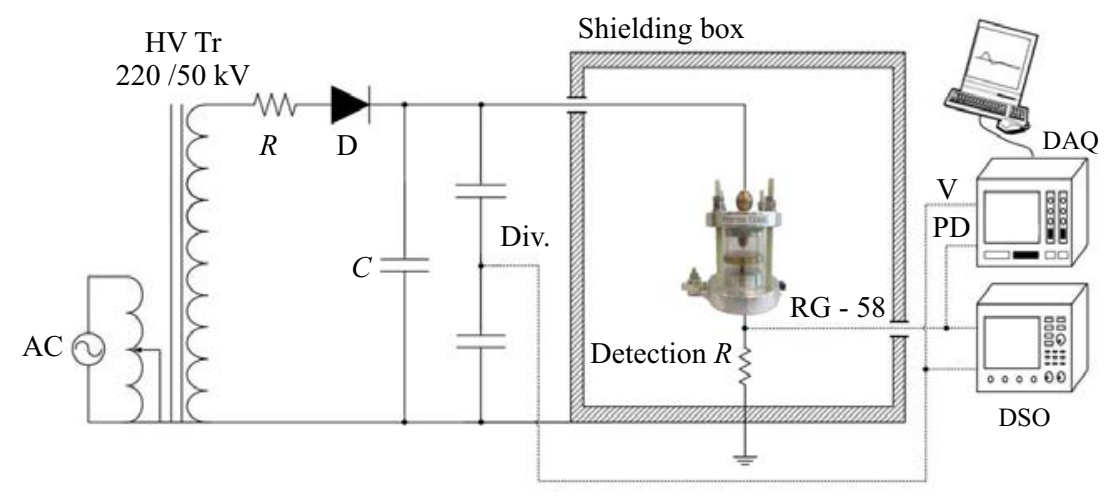

Fig. 1. Experimental setup 

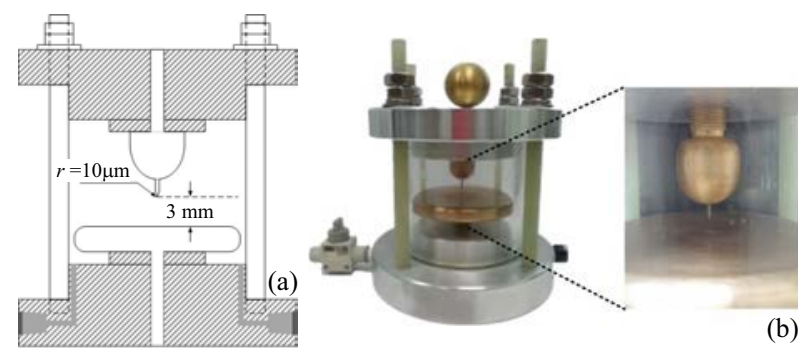

Fig. 2. Insulation defect of protrusion on conductor: (a)-schematic; (b) - photograph

maximum output of $50 \mathrm{kV}$ and $30 \mathrm{~mA}$ was PD-free. It was immersed in insulation oil to ensure that there was no corona occurring adjacent to the high voltage connection. A resistor $R$ was used in the test circuit for limiting the current that may damage the measuring instruments. Artificial defects filled with SF6 gas were placed inside a shielding box to reduce the external interference $[3,11]$.

The applied voltage was measured by a high-voltage capacitive divider (Div., North Star High Voltage, VD100). For accurate measurement, PD signals generated from the artificial defects were detected through a $50 \Omega$ non-inductive resistor that was connected between the defect and the ground. A digital storage oscilloscope (DSO, Yokogawa, DL9140) with a sampling rate of $5 \mathrm{GS} / \mathrm{s}$ and a bandwidth of $1 \mathrm{GHz}$, as well as a data acquisition unit (DAQ, National Instruments, NI-5114) with a sampling rate of $250 \mathrm{MS} / \mathrm{s}$ and a bandwidth of $50 \mathrm{MHz}$ were used for signal acquisition. To avoid an electric potential difference, the transformer, voltage divider, artificial defects, and measuring instruments were grounded.

PD occurs at insulation defects generated during the manufacture, assembly, and transportation procedures of GIS, where the local electrical stress is higher than the insulation strength. Most of such defects can be detected in the factory test or commissioning test, whereas there are still some micro ones hard to be found. Left without checking, these defects cause progressive deterioration of the dielectric material even though facilities operate at their rated voltage. Since PD presents different patterns depending on the type of insulation defects, it is necessary to study their specific characteristics. Typical insulation defects in GIS include protrusion on conductor (POC), protrusion on enclosure (POE), free particle (FP), and crack inside spacer (CIS). In this paper, four artificial defects were fabricated. They were filled with $0.5 \mathrm{MPa}$ SF6 with a purity of $99.99 \%$. To avoid any pollution of SF6, a vacuum pump was used to vacuumed the defects for 30 minutes before gas injection. Figure 2 shows the schematic and photograph of POC.

In the POC and POE, a needle electrode with a curvature radius of $10 \mathrm{~m}$ and a plane electrode with a diameter of $80 \mathrm{~mm}$ were used. The distance between two electrodes was $3 \mathrm{~mm}$. The needle electrode was used to represent a micro-size metallic protrusion on the conductor or chamber of gas insulated structures. The plane electrode was made of tungsten copper and its edge was rounded to prevent corona occurring due to the concentration of electric field. The FP, which is the most common defect, was fabricated with a $1 \mathrm{~mm}$-diameter aluminum sphere to simulate a free moving metallic particle. The CIS was designed to simulate deficiency in the spacer of gas insulated structures that may result from mechanical impact. It was fabricated using an epoxy insulation plate with a diameter of $80 \mathrm{~mm}$ and a thickness of $5 \mathrm{~mm}$.

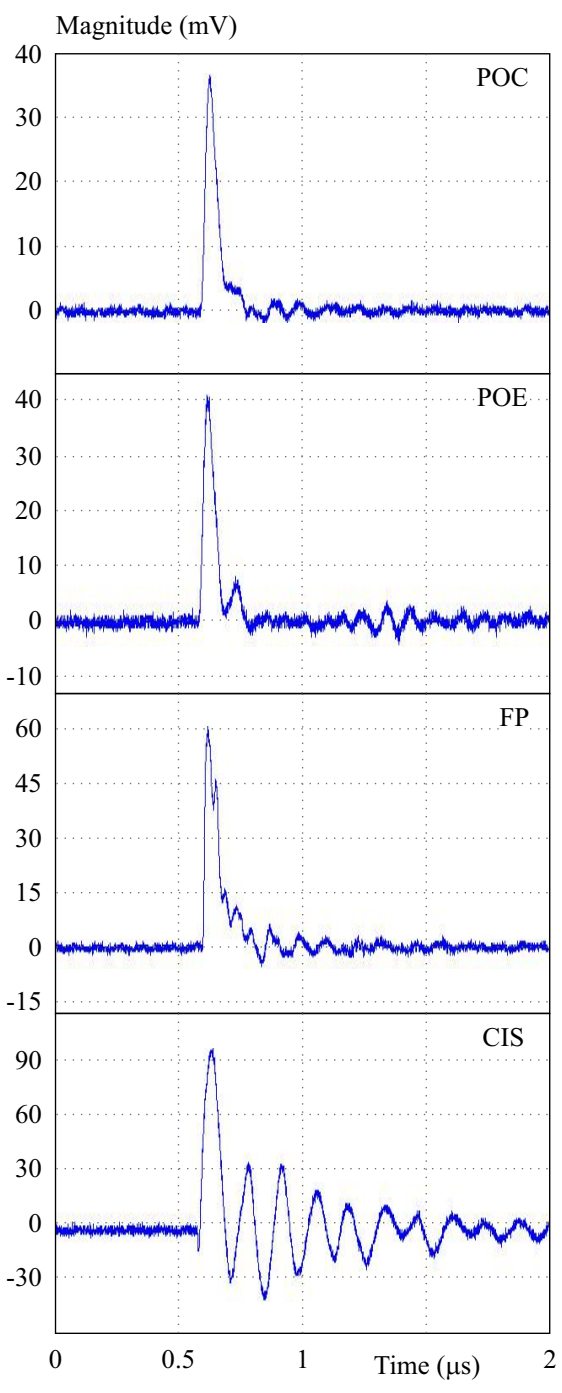

Fig. 3. Single PD pulses

\section{Methods and results}

\subsection{PD Signal and noises}

As shown in Fig. 3, PD presented as a damped exponential pulse (DEP) in the POC, POE as well as FP, and presented as a damped oscillatory pulse (DOP) in the CIS. The DEP-type pulse had an average rise time of $16 \mathrm{~ns}$, an average decay time of $60 \mathrm{~ns}$, and an average pulse width of $52 \mathrm{~ns}$. The rise time, decay time, and pulse width of DOP- type pulse were $30 \mathrm{~ns}, 35 \mathrm{~ns}$, and $65 \mathrm{~ns}$, respectively.

During on-site PD monitoring, pulse sequences rather than single pulse are acquired for evaluating the severity of insulation defect, for extracting discharge features 

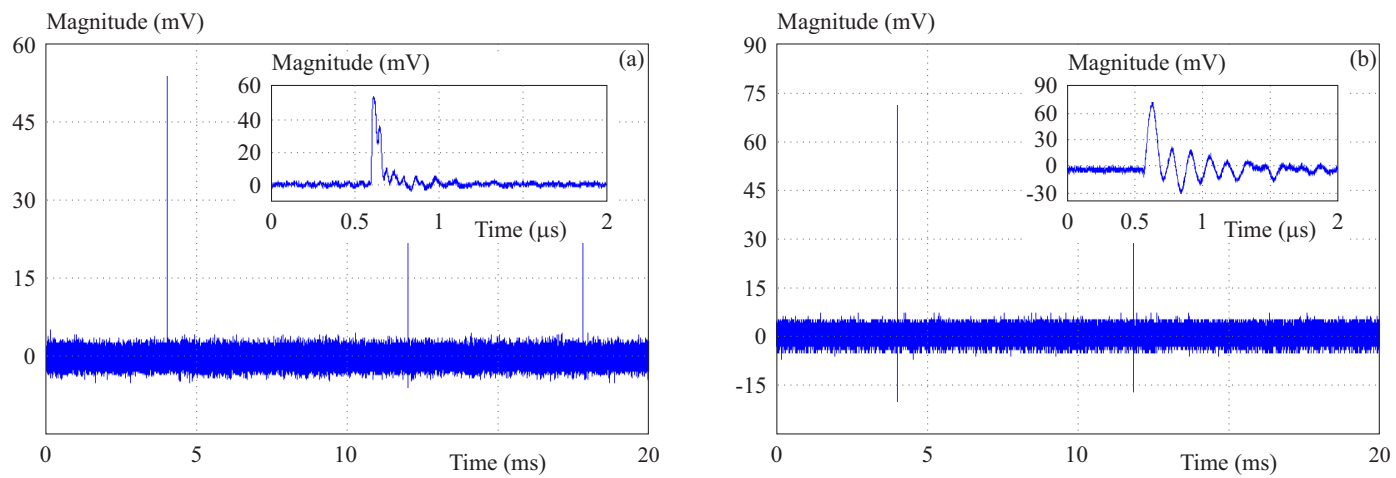

Fig. 4. PD pulse sequence: (a) - damped exponential pulse type, (b) - damped oscillatory pulse type

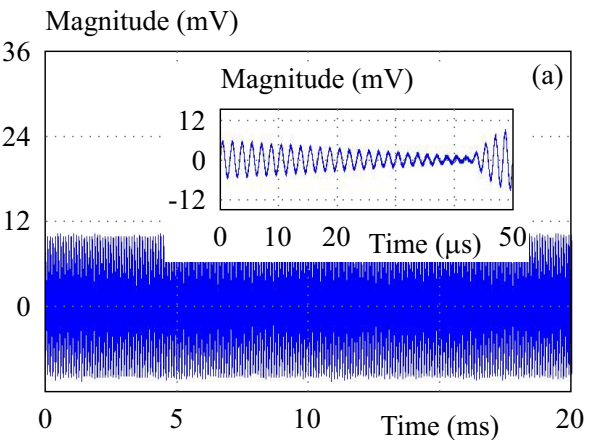

Magnitude (mV)

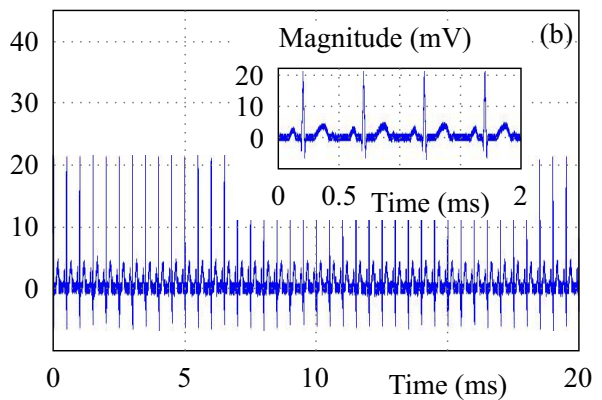

Magnitude (mV)

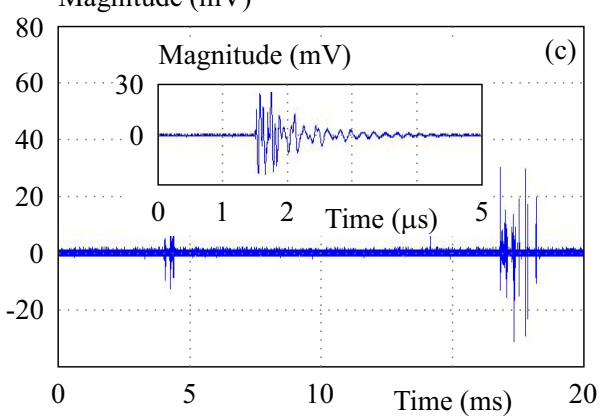

Fig. 5. Noises in PD detection. (a) Amplitude modulation (AM) radio interference; (b) periodic non-sinusoidal noise; and (c) switching impulse

and finally for classifying the defect type. Therefore, the WT techniques were used to discriminate practical PD pulse sequences and the effectiveness was compared with a high-pass filter that had a cutoff frequency of $800 \mathrm{kHz}$. A DEP-type PD sequence and a DOP-type PD sequence that were interfered by background noise, AM radio interference, non-sinusoidal noise, and switching impulse were used to verify the effect of wavelet de-nosing. Figure 4 shows the two types of PD pulse sequence, which inherently contained the background noise generated from the detection system.

Figure 5 shows noises commonly presented in PD detection. The noises were also acquired in $20 \mathrm{~ms}$ and detail information was indicated in the inset graph. AM radio signal with a central frequency of $600 \mathrm{kHz}$ and a magnitude of $10 \mathrm{mV}$ generated from a signal generator was externally injected to the PD pulse sequence to present the DSI. Figure 5(b) is a periodic non-sinusoidal noise with main component of $2 \mathrm{kHz}$ to simulate interference from power electronics [23]. A switching impulse shown in Figure 5(c) that had a pulse width higher than that of PD pulse was used to represent the stochastic pulse-shaped interferences.

\subsection{LabVIEW based wavelet transform}

The proposed wavelet de-noising method was developed based on LabVIEW program. The virtual instruments (VI) block diagram is shown in Figure 6, which is mainly composed of discrete wavelet transformation VI and VIs of threshold, thresholding function as well as de-noising evaluation. The interfered signal was first decomposed into 8 levels using bior2.6 mother wavelet by discrete wavelet transformation, generating the detail (D) coefficients at all levels and the approximation coefficients (A) at the highest level. In the loop, the detail coefficients at each decomposition level were extracted respectively and then modified by the medium thresholding function and automatic threshold. A shift register was used to pass detail coefficients from previous iteration through the loop to the next iteration. Finally, the modified detail coefficients and the original approximation coefficients were used for signal reconstruction by the inverse discrete wavelet transformation. Effectiveness of de-noising was verified by the evaluation VI, in which the correlation coefficient $(\gamma)$, reduction in noise $(\mathrm{RN})$, and change in amplitude (A\%) were calculated [19, 20, 24-26].

The correlation coefficient that is given by

$$
\gamma=\frac{\sum_{i=1}^{N-1}[X(i)-\bar{X}][Y(i)-\bar{Y}]}{\sqrt{\sum_{i=1}^{N-1}[X(i)-\bar{X}]^{2} \sum_{i=1}^{N-1}[Y(i)-\bar{Y}]^{2}}}
$$




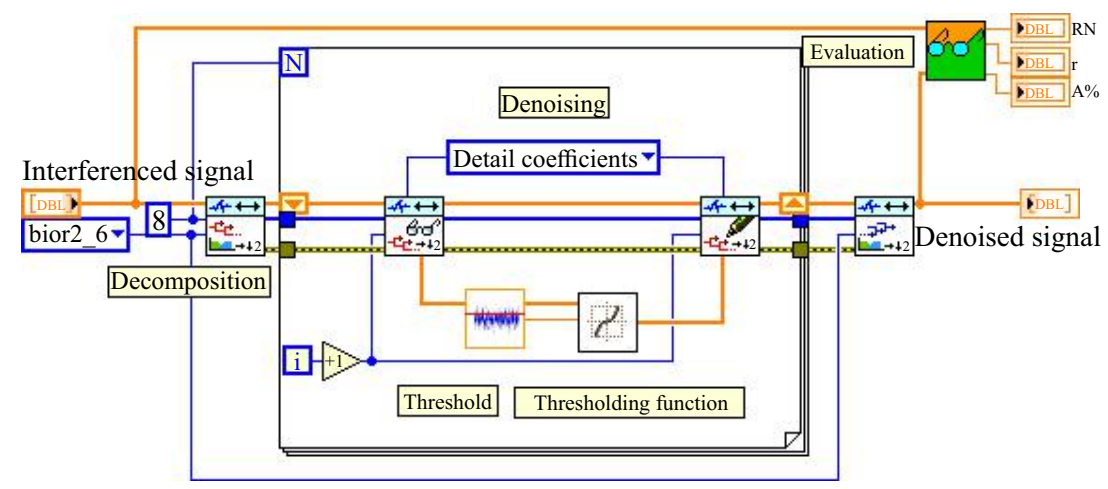

Fig. 6. VI block diagram of wavelet de-noising
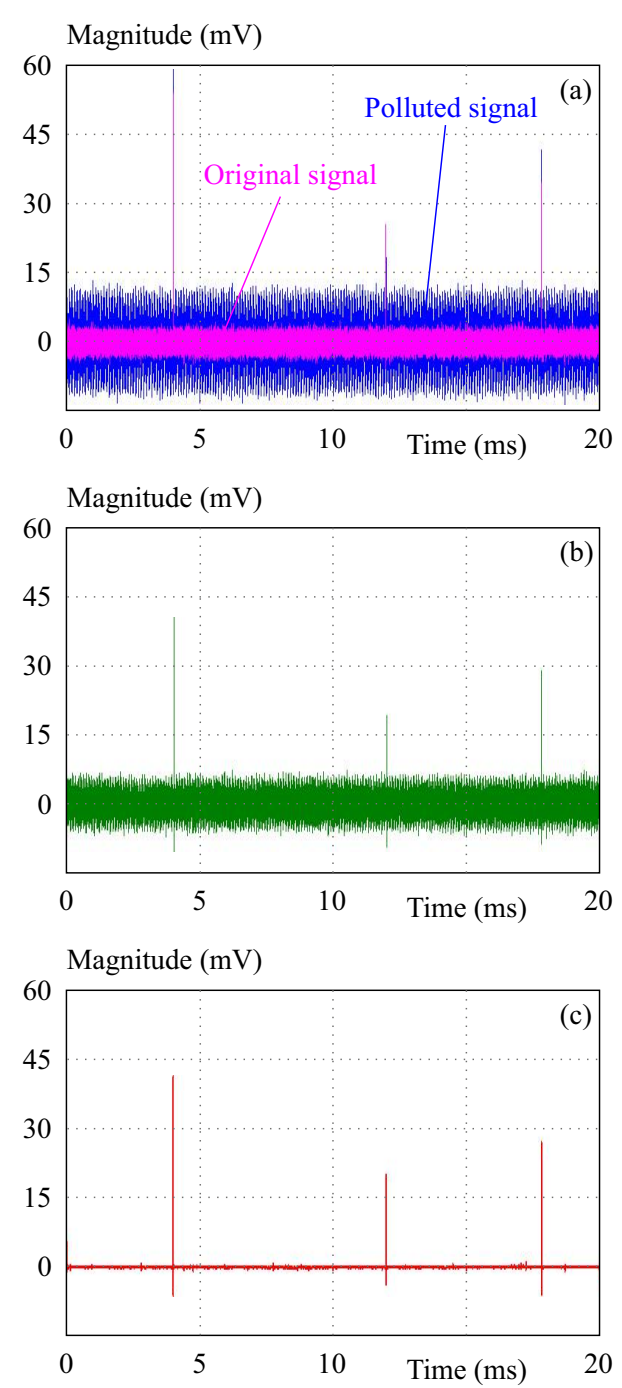

Fig. 7. Discrimination of DEP-type pulse interfered by AM interference: (a) - original signal and interfered signal, (b) de-noised by filter, and (c) - de-noised by wavelet method

where $X(i)$ and $Y(i)$ are the original PD pulse sequence and the de-noised signal, respectively, and $\bar{X}$ and $\bar{Y}$ are their mean values. A larger value of correlation coefficient indicates a higher similarity between these two signals. The RN and $\mathrm{A} \%$ are defined as

$$
R N=10 \log \sum_{i=0}^{N}[X(i)-Y(i)]^{2}, A \%=100 \frac{|X-Y|}{X}
$$

where $X$ and $Y$ are peak values of the original signal and the de-noised signal, respectively. After de-noising by WT method, noise should be eliminated effectively while the original signal should not be distorted. Therefore, an acceptable de-noising method corresponds to the large values of $\gamma$ and $\mathrm{RN}$, as well as the low value of $\%$.

\subsection{De-noising of Damped Exponentional Pulse Se- quence}

Figure 7 shows discrimination of DEP-type pulse sequence interfered by AM interference using the high-pass filter with cutoff frequency of $800 \mathrm{kHz}$ and the wavelet method. In Figure 7(a), the original signal contained three PD pulses that had different magnitudes. Such signal was interfered by AM interference with central frequency of $600 \mathrm{kHz}$. It can be seen that the original signal was immersed in the noise and it is hard to distinguish the second discharge pulse from the interfered signal by visual inspection. Figure 7(b) and 7(c) show de-noising of the interfered signal using high-pass filter and wavelet method, respectively. After de-noising by high-pass filter, the background noise and AM interference were not completely eliminated. As a result, the de-noised signal had a correlation coefficient of 0.07 with the original signal, a reduction in noise of $6.65 \mathrm{~dB}$, and a change in amplitude of $25.21 \%$. On the contrary, both the background noise and $\mathrm{AM}$ interference were significantly reduced by the wavelet method. Details of the application of medium thresholding function-automatic threshold to the detail coefficients and the signal reconstruction are illustrated in Fig. 8. Detail coefficients that were related with $\mathrm{PD}$ were retained whereas that associated with noise were suppressed. The de-noised signal was reconstructed by all of the modified detail components D1D8 and the original approximation component A8. The correlation coefficient, reduction in noise, and change in amplitude values of signal de-noised by wavelet method were $0.17,11.64 \mathrm{~dB}$, and $23.36 \%$, respectively, all of which showed the superiority compared with the high-pass filter method.

Table 1 shows the summary of discriminating DEP and DOP type sequence. From the correlation coefficient, reduction in noise, and change in amplitude, it revealed that the wavelet method had higher performance compared with the high-pass filter method for eliminating the 
Table 1. Results of discriminating DEP \& DOP pulse sequence

\begin{tabular}{lccccccc}
\hline \multirow{2}{*}{ Method } & \multicolumn{3}{c}{ DEP } & \multicolumn{3}{c}{ DOP } \\
& Evaluation & $\begin{array}{c}\text { Amplitude } \\
\text { modulation }\end{array}$ & $\begin{array}{c}\text { Non-sinusoidal } \\
\text { noise }\end{array}$ & $\begin{array}{c}\text { Switching } \\
\text { impulses }\end{array}$ & $\begin{array}{c}\text { Amplitude } \\
\text { modulation }\end{array}$ & $\begin{array}{c}\text { Non-sinusoidal } \\
\text { noise }\end{array}$ & $\begin{array}{c}\text { Switching } \\
\text { impulses }\end{array}$ \\
\cline { 3 - 8 } Wavelet & Correlation $-\gamma$ & 0.17 & 0.17 & 0.20 & 0.17 & 0.17 & 0.21 \\
& Noise reduction $(\mathrm{dB})$ & 11.64 & 0.71 & -7.14 & 6.03 & 1.87 & -4.15 \\
& Amplitude change(\%) & 23.36 & 27.67 & 13.89 & 22.41 & 24.90 & 15.58 \\
\hline \multirow{3}{*}{ Filter } & Correlation- $\gamma$ & 0.07 & 0.13 & 0.15 & 0.10 & 0.16 & 0.15 \\
& Noise reduction $(\mathrm{dB})$ & 6.65 & 0.19 & -12.72 & 4.55 & 1.04 & -8.81 \\
& Amplitude change $(\%)$ & 25.21 & 29.41 & 18.24 & 24.15 & 28.27 & 20.11 \\
\hline
\end{tabular}
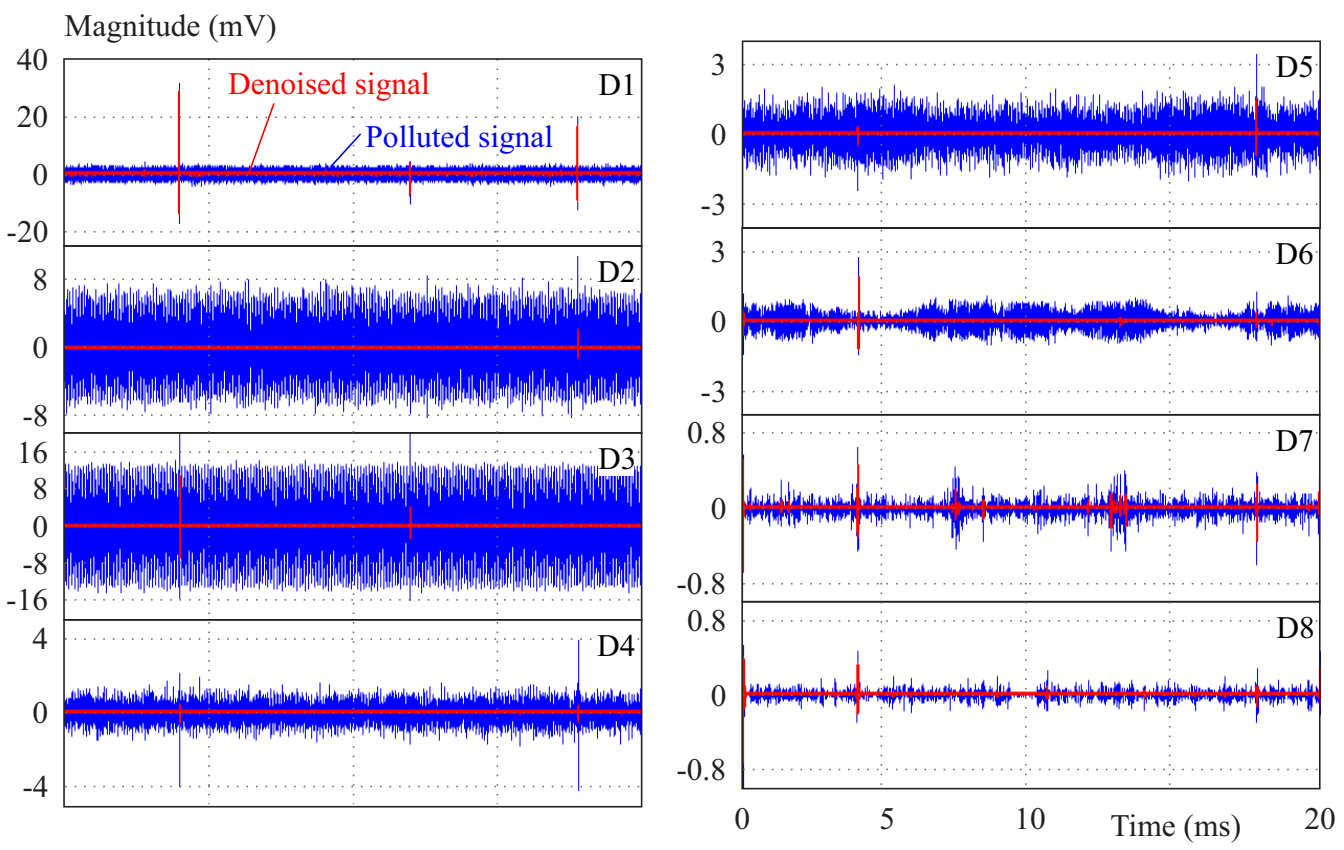

Fig. 8. Elimination of AM interference from DEP and DOP type pulse sequence by wavelet method
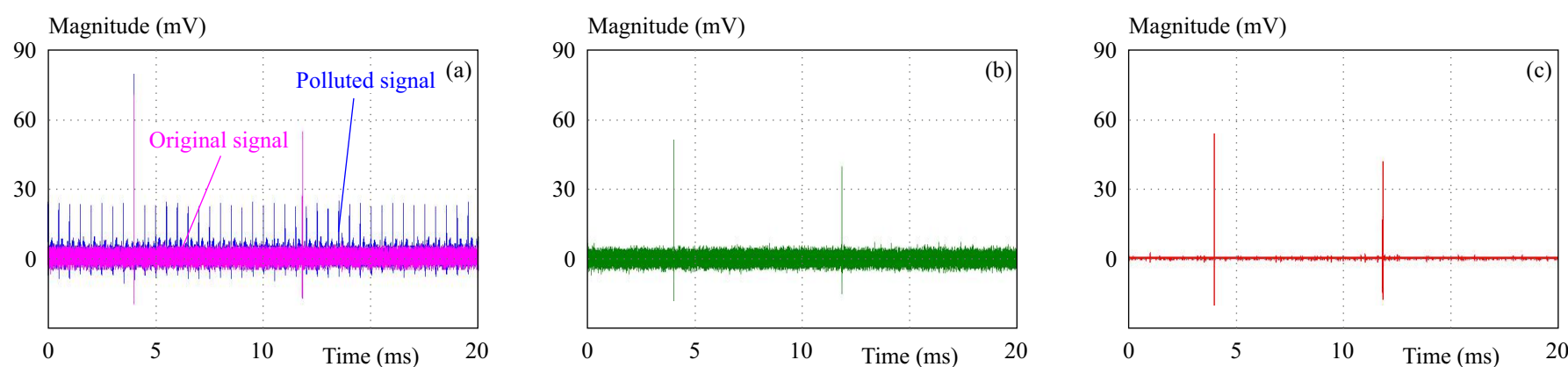

Fig. 9. Discrimination of DOP-type pulse interfered by non-sinusoidal noise: (a) - original signal and interfered signal, (b) - de-noised by filter, and (c) - de-noised by wavelet method

background noise, AM radio interference, non-sinusoidal noise, and switching impulse.

\subsection{De-noising of damped oscillatory pulse sequence}

Figure 9 demonstrates the elimination of periodic non-sinusoidal noise from interfered DOP-type pulse sequence. In Figure 9(a), the non-sinusoidal noise had main component with frequency of $2 \mathrm{kHz}$ and magnitude of 20 $\mathrm{mV}$ and other components with magnitudes lower than $20 \mathrm{mV}$. It can be seen that the first PD pulse with magnitude of $71 \mathrm{mV}$ merged with the noise pulse, resulting in an increase in the peak magnitude of pulse sequence. This case may lead to an overvaluation of the PD severity and cause an erroneous diagnosis result. The result of denoising using the high-pass filter is shown in Figure 9(b). 
Magnitude $(\mathrm{mV})$

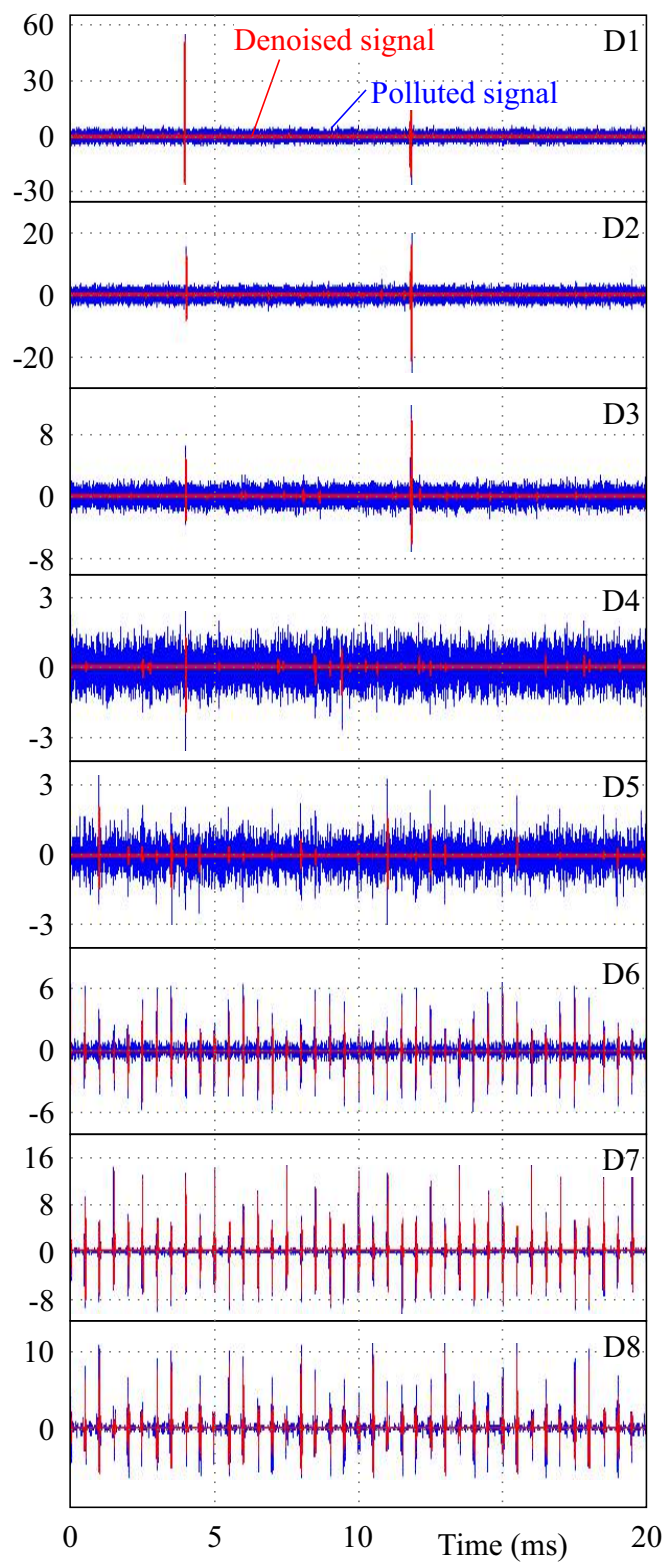

Fig. 10. Elimination of non-sinusoidal noise from DOP-type pulse sequence by wavelet method

Applying the high-pass filter suppressed the periodic nonsinusoidal, however, there were still remained interference in the de-noised signal. The correlation coefficient, reduction in noise, and change in amplitude of filter method were $0.16,1.04 \mathrm{~dB}$, and $28.27 \%$, respectively. Figure 10 illustrates the original detail components of interfered signal and the modified detail components that were denoised by bior2.6 mother wavelet, medium thresholding function, and automatic threshold. As de-noising of DEPtype pulse sequence interfered by non-sinusoidal noise, most of PD-related components were observed in detail components D1-D5, they were therefore de-noised and then used for signal reconstruction.

Although de-noised by wavelet method, the detail components D6-D8 were found to be related with background noise and periodic non-sinusoidal noise, therefore, they were discarded instead of being used for signal reconstruction. Figure 9(c) shows the de-noised signal obtained by wavelet method. The correlation coefficient was 0.17 . The reduction in noise and change in amplitude were $1.87 \mathrm{~dB}$ and $24.90 \%$, respectively. The results of discriminating DOP-type pulse sequence can be compared in Tab. 1. Discrimination of PD signal under HVDC using wavelet techniques resulted in higher values of correlated coefficient as well as reduction in noise, and lower value of change in amplitude compared with the highpass filter. The wavelet method showed its effectiveness in de-noising DOP-type PD sequence that was interfered by background noise, amplitude modulation radio interference, non-sinusoidal noise, and switching impulse.

\section{Conclusions}

This paper dealt with discrimination of PD signals interfered by noises and interferences using wavelet transform for on-line condition monitoring and diagnosis of HVDC GIS, aiming to improve the detection sensitivity and further the accuracy of severity assessment. The wavelet techniques were used for discriminating DEPtype and DOP-type PD sequences that were actually detected from artificial defects and interfered by background noise, amplitude modulation radio interference, periodic non-sinusoidal noise, and switching impulse. Although the PD pulses were immersed in the noises and cannot be distinguished by visual inspection, they can be recovered after applying wavelet de-noising. De-noising with the wavelet method resulted in an average reduction in noise of $1.49 \mathrm{~dB}$, correlation coefficient of 0.18 , and change in amplitude of $21.30 \%$, all of which showed the superiority of wavelet method compared with the highpass filter method. The proposed wavelet techniques were verified to be effective in discriminating $P D$ signals from noises and interferences. Results from this paper were expected to be applied for insulation diagnosis of HVDC GIS, by which accuracies of PD detection, risk assessment, defect identification and localization can be significantly improved.

\section{REFERENCES}

[1] P. Gill, Electrical Power Equipment Maintenance and Testing, 2nd ed., CRC Press, New York, United State, pp. 1-18, 2008.

[2] M. G. Danikas and F. V. Topalis, "Partial Discharge Considerations Gas Insulated Switchgear (GIS)", Journal of Electrical Engineering, vol. 53, no. 9, pp. 281-284, 2000.

[3] G. M. Wang and G. S. Kil, "Measurement and Analysis of Partial Discharge using an Ultra-high Frequency Sensor for Gas Insulated Structures", Metrology andMeasurement Systems, vol. 24, no. 3, pp. 515524, 2017.

[4] CIGRE Working Groups, Risk Assessment on Defects GIS Based on PD Diagnosis; CIGRE: Paris, France, 2013.

[5] International Electrotechnical Commission, High-Voltage Test Techniques-Partial Discharge Measurements; IEC 60270; IEC: Geneva, Switzerland, 2000. 
[6] G. M. Wang, S. J. Kim, G. S. Kil, and S. W. Kim, "Optimization of Wavelet and Thresholding for Partial Discharge Detection under HVDC", IEEE Transactions on Dielectrics Electrical Insulation, vol. 24, no. 1, pp. 200208, 2017.

[7] S. Ramanujam, P. D. Singh, and M. G. Danikas, "Characterization of Partial Discharge Transformer Oil Insulation under AC and DC Voltage using Acoustic Emission Technique", Journal of Electrical Engineering, vol. 58, no. 2, pp. 91-97, 2007.

[8] S. Tenbohlen, D. Denissov, S. M. Hoek, and S. M. Markalous, "Partial Discharge Measurement the Ultra High Frequency (UHF) Range", IEEE Transactions on Dielectrics Electrical Insulation, vol. 15, no. 6, pp. 1544-1552, 2008.

[9] M. Wu, H. Cao, J. Cao, H. L. Nguyen, J. B. Gomes, and S. P. Krishnaswamy, "An Overview of State-of-the-Art Partial Discharge Analysis Techniques for Condition Monitoring", IEEE Electrical Insulation Magazine, vol. 31, no. 6, pp. 2235, 2015.

[10] M. D. Judd, L. Yang, and I. B. B. Hunter, "Partial Discharge Monitoring for Power Transformers using UHF Sensors Part 1: Sensors and Signal Interpretation", IEEE Electrical Insulation Magazine, vol. 21, no. 2, pp. 5-14, 2005.

[11] G. M. Wang, H. E. Jo, S. J. Kim, S. W. Kim, and G. S. Kil, "Measurement and analysis of partial discharges SF6 gas under HVDC", Measurement, vol. 91, pp. 351359, 2016.

[12] P. H. F. Morshuis and J. J. Smit, "Partial Discharges at dc Voltage: Their Mechanism, Detection and Analysis", IEEE Transactions on Dielectrics Electrical Insulation, vol. 12, no. 2, pp. 328-340, 2005.

[13] R. Piccin, A. R. Mor, P. Morshuis, A. Girodet, and J. Smit, "Partial Discharge Analysis of Gas Insulated Systems at High Voltage AC and DC", IEEE Transactions on Dielectrics Electrical Insulation, vol. 22, no. 1, pp. 218-228, 2015.

[14] F. H. Kreuger, Partial Discharge Detection High-Voltage Equipment, 1st ed., Butterworths, London, United Kingdom, pp. 1-152, 1990.

[15] A. Haddad and D. F. Warne, Advances High Voltage Engineering, 1st ed., The Institution of Engineering and Technology, Stevenage, UK, pp. 3773, 2004.

[16] P. Widger and A. Haddad, "Evaluation of SF6 Leakage from Gas Insulated Equipment on Electricity Networks Great Britain", Energies, vol. 11, no. 8, pp. 2037(1)-(14), 2018.

[17] International Electrotechnical Commission, "High-Voltage Switchgear and Controlgear-Part 203: Gas-Insulated Metal-Enclosed Switchgear for Rated Voltages above 52 kV", IEC 62271-203, IEC, Geneva, Switzerland, 2011.

[18] H. Zhang, T. R. Blackburn, B. T. Phung, and D. Sen, "A Novel Wavelet Transform Technique for On-line Partial Discharge Measurements Part 1: WT De-nosing Algorithm", IEEE Transactions on Dielectrics Electrical Insulation, vol. 14, no. 1, pp. 3-14, 2007.

[19] L. Satish and B. Nazneen, "Wavelet-based Denoising of Partial Discharge Signals Buried Excessive Noise and Interference", IEEE Transactions on Dielectrics Electrical Insulation, vol. 10, no. 2, pp. 354367, 2003.

[20] G. M. Wang, G. S. Kil, H. K. Ji, and J. H. Lee, "Disturbance Elimination for Partial Discharge Detection the Spacer of Gas-insulated Switchgears", Energies, vol. 10, no. 11, pp. 1-12, 2017.

[21] X. Ma, C. Zhou, and I. J. Kemp, "Interpretation of Wavelet Analysis and Its Application Partial Discharge Detection", IEEE Transactions on Dielectrics Electrical Insulation, vol. 9, no. 3, pp. 446457, 2002.

[22] X. Zhou, C. Zhou, and I. J. Kemp, “An Improved Methodology for Application of Wavelet Transform to Partial Discharge Measurement Denoising", IEEE Transactions on Dielectrics Electrical Insulation, vol. 12, no. 3, pp. 586594, 2005.

[23] O. A. Giddani, A. Y. M. Abbas, G. P. Adamb, O. Anaya-Lara, and K. L. Lo, "Multi-task Control for VSCHVDC Power and
Frequency Vontrol", International Journal of Electrical Power and Energy Systems, vol. 53, pp. 684-690, 2013.

$24]$ B. Moula, A. Mekhaldil, M. Teguar, and A. Haddad, "Characteristics of Discharge on Non-uniformly Polluted Glass Surface using a Wavelet Transform Approach", IEEE Transactions on Dielectrics Electrical Insulation, vol. 20, no. 4, pp. 1457-1466, 2013.

25] I. Shim, J. J. Soraghan, and W. H. Siew, "Detection of PD Utilizing Digital Signal Processing Method. Part 3: Open-Loop Noise Reduction", IEEE Electrical Insulation Magazine, vol. 17, no. 1, pp. 613,2001

26] J. Li, T. Jiang, S. Grzybowski, and C. Cheng, "Scale Dependent Wavelet Selection for De-noising of Partial Discharge Detection", IEEE Transactions on Dielectrics Electrical Insulation, vol. 17 , no. 6 , pp. 1705-1714, 2011.

Received 20 April 2020

Guoming Wang received the MEng degree and $\mathrm{PhD}$ degrees from Division of Electrical and Electronics Engineering, Korea Maritime and Ocean University, Korea in 2015 and 2019, respectively. He is currently a researcher at Hangzhou Guozhou Power Technology Co., Ltd. His research interests are in the area of high voltage engineering, and diagnosis of power facilities.

Han $\mathrm{Hu}$ received the BEng degree from Division of Electronic and Information, Hangzhou Dianzi University, China in 2005. He is currently a senior engineer at Hangzhou Guozhou Power Technology Co., Ltd. His research interests are condition monitoring and diagnosis of power facilities, high voltage engineering, and asset management.

Min Tang received the BEng degree from Division of Electronic and Information, Hangzhou Dianzi University, China in 2006. He is currently a senior engineer at Hangzhou Guozhou Power Technology Co., Ltd. His research interests are condition monitoring and diagnosis of power facilities and asset management.

Jinlong Gong received the MEng degree from Division of Electrical Engineering, Zhejiang University, China in 2006. He is currently a senior engineer at State Grid Zhejiang Electric Power Research Institute. He joined IEEE China PES Committees in 2019. His research interests are diagnostics of insulation systems by partial discharge analysis and calibration of high-voltage instrument.

Yifan Wang received the MEng degree from Division of Power System Engineering, University College London, The United Kingdom in 2013. He is currently a senior engineer at State Grid Zhejiang Electric Power Research Institute. His research interests are diagnostics of insulation systems by partial discharge analysis and inspection of transformers.

Hong-Keun Ji received the BEng, MEng, and PhD degree from Division of Electrical and Electronics Engineering, Korea Maritime and Ocean University, Korea in 2008, 2010, and 2018, respectively. He is currently a forensic researcher at National Forensic Service Daegu Institute. His research interests are in the area of diagnosis of power facilities.

Gyung-Suk Kil (Professor) received the BEng, MEng, and PhD degrees from Inha University, Korea in 1984, 1987, and 1996, respectively. Since then, he has been a professor at the Division of Electrical and Electronics Engineering, Korea Maritime and Ocean University. He worked as a visiting professor at Cardiff University in 2003. His research interests are in the area of high voltage engineering and diagnosis of power facilities. 